\title{
Drivers and their influences on variation of aboveground carbon removals in miombo woodlands of mainland Tanzania
}

Bernardol John Manyanda ( $\sim$ bernardoljohnm@gmail.com )

Sokoine University of Agriculture https://orcid.org/0000-0001-8796-2752

Emmanuel Fred Nzunda

Sokoine University of Agriculture Faculty of Forestry and Nature Conservation

Wilson Ancelm Mugasha

Sokoine University of Agriculture Faculty of Forestry and Nature Conservation

Rogers Ernest Malimbwi

Sokoine University of Agriculture Faculty of Forestry and Nature Conservation

\section{Research}

Keywords: Drivers, aboveground carbon, emissions, miombo, removals

Posted Date: May 8th, 2020

DOl: https://doi.org/10.21203/rs.3.rs-18917/v1

License: (c) (i) This work is licensed under a Creative Commons Attribution 4.0 International License. Read Full License 


\section{Abstract \\ Background}

Removals caused by both natural and anthropogenic drivers such as logging and fire causes substantial carbon emissions. Better insights into drivers and their variations of aboveground carbon removals is therefore needed. We assessed the drivers of aboveground carbon (AGC) removals and quantified the dynamics of removals-induced carbon emissions due to drivers using the National Forest Resources Assessment and Monitoring (NAFORMA) data sets in R software. Miombo woodlands which is the largest forest formations covering about $93 \%$ of forest land in mainland Tanzania was the case study.

\section{Results}

Drivers of AGC removals in miombo woodlands of mainland Tanzania in order of importance were; timber, fire, shifting cultivation, charcoal, natural death, firewood collection, poles, grazing by wildlife animals, carvings, grazing by domestic animals, and mining. The average AGC removals by drivers range from $0.0-1.273 \mathrm{tCha}^{-1}$ year $^{-1}$.

\section{Conclusions}

Increased mitigation efforts in addressing removals by timber, fires, shifting cultivation, charcoal and natural death would be effective in addressing forest degradation in the REDD + process in Tanzania. Since NAFORMA provides national picture on drivers and their variation on AGC removals, site-specific studies need to be conducted to bring information that would be used for local forest management. This kind of study need to be conducted in other vegetation types like Montane and Mangrove forest in Tanzania.

\section{Background}

Managing the carbon stocks of the land use sector is currently a key focus for climate change mitigation in developing countries $[1,2,3]$. In terrestrial ecosystems, forests and woodlands play a major role for the mitigation and adaptation to climate change via carbon storage [4, 2]. After oceans, forests are the world's largest storehouses of carbon and they provide ecosystem services that are important to human wellbeing [5]. Tropical forests alone store a quarter of a trillion tons of carbon in above and below ground biomass [6]. Notwithstanding their contribution to the climate change mitigation, Tanzania's forests face enormous challenges including deforestation and forest degradation [7].

Deforestation and forest degradation are amongst the major anthropogenic sources of greenhouse gas emissions (GHG), contributing about 17 per cent globally [8]. Of the total emissions, degradation is responsible for at least one-fifth in the Brazilian Amazon [9], two-thirds in Indonesian forests [10], and 
almost half in African tropical forests [11]. Forest degradation also leads to forest fragmentation and can contribute to deforestation [12]. While deforestation refers to a permanent or long-term conversion of forest to non-forest land $[13,14]$, forest degradation is the changes within the forest that negatively affect the structure or function of the stand and/or site, and thereby lower the capacity to supply products and/or services $[15,16]$.

The changes within the forests involves removals of trees and hence contributing to carbon emissions. The drivers of carbon removals are multifaceted and cannot be reduced to a few variables; rather they operate at different levels and scales in the human-environment linkage [17]. These drivers are divided into two broad categories: proximate and underlying causes. Proximate causes are typically human activities operating at the local level. They include shifting cultivation and cattle ranching, wood extraction through logging or charcoal production, and infrastructural development such as transportation, markets and settlements. On the other hand, underlying causes do not directly cause removals but influence the proximate causes. This category includes a complexity of economic issues, policies and institutions, technological factors, socio-cultural, and demographic factors $[11,17,18]$.

Aboveground biomass (AGB) is not static, but rather spatially and temporally highly variable, particularly in the tropics with the same factor likely having different results $[19,20,21]$. This makes its quantification challenging. It is generally assumed that about half of AGB consists of carbon in different vegetation types including miombo woodlands in Tanzania. Miombo woodlands are the largest vegetation types in Tanzania covering about $93 \%$ of the forest area of 48.1 million ha [22]. As in other tropical forest landscape, complex matrices of low to high AGC removal densities can be expected in entire miombo woodlands in Tanzania and its management categories due to varying drivers. Additionally, which drivers contribute more to the variations of AGC removals in the entire miombo woodlands and its management categories is to a large extent unknown. This has been due to lack of appropriate assessment mechanism. Nevertheless, the Mainland Tanzania National Forest Inventory (NFI) data source which is commonly referred as NAFORMA, have recently become available based on country REDD + readiness activities that allow assessment of AGC removals and their amount of AGC emissions in miombo woodlands [3]. The objective of the present study was to identify the drivers of AGC removals and assess which of the identified drivers contribute more to the variation of AGC in miombo woodlands of Tanzania mainland. Specifically the study sought to: (1) identify drivers of AGC removals (2) quantify the amount of AGC removals by each driver and, (3) Ranking the identified drivers in order of their contribution to the variations of $A G C$.

Understanding the drivers of AGC removals and their amount of AGC removed is fundamental for better design of REDD + strategy. In some cases, REDD + incentives would be channeled directly to affect drivers. Moreover, a better understanding of drivers of AGC removals are required as part of developing mitigation interventions at sub-national levels to ensure improved land-use change. This kind of understanding is also crucial for subsequent development of management plans in order to tackle each driver in response to the amount of AGC emissions caused. 


\section{Results}

\section{Drivers and their corresponding number of stems and aboveground carbon removals}

We identified eleven drivers for tree cutting and these were, forest fires, firewood collection, grazing by both wildlife, domesticated animals, carving, poles, shifting cultivation, timber, and mining activities (Table 1).

\section{Drivers and their variations on the number of stems and AGC removals}

Table 1 also shows the contribution of the drivers in terms of the number of stems and AGC removals per hectare per year for miombo woodlands in Tanzania. Higher number of stems/ha/year were removed by shifting cultivation, followed by charcoal, natural death, firewood collection and poles. In terms of biomass however, we observed higher AGC removals by timber followed by fire, shifting cultivation, charcoal and natural death (Table 1).

The contribution of the drivers with regards to number of stems and carbon removals were further expressed on the basis of different management categories and subcategories of miombo woodlands. Considering Tanzania Forest Services Agency (TFS) administrative zones, large number of stems were removed by charcoal followed by firewood collection and shifting cultivation whereas grazing was the least in the central zones (Table 2). Onn the other hand, charcoal removed more AGC followed by firewood collection, natural death and shifting cultivation while grazing had the least removals. In the other zones, the drivers seem to change leading positions between charcoal production, timber and fire (Table 2).

Considering vegetation types, natural death, timber production and shifting cultivation appear to be leading causes of removals interchangeably in the closed woodlands, open woodlands and Woodlands with scattered cropland for both number of stems and AGB (Table 3). Grazing, mining and carvings are among the least contributors to removals in the three vegetation types. Regarding ownership types, higher number of stem removals were observed due to natural death followed by fire, poles and timber in the central government land (Table 4). While the least number of stems removals per hectare per year was observed due to carving followed by grazing domestic and charcoal. The highest AGC was removed as timber followed by natural death, fire and charcoal. Carvings, grazing domestic and shifting cultivation accounted for the least AGC removals in this ownership types (Table 4). The contribution of the drivers of removal in terms of number of stems and AGC removals appear to be changing leading positions in other ownership types i.e. general land, local government land, private land and village land (Table 4).

Table 5 indicates drivers and the variations of number of stem and carbon removals in the different land use types. Regarding protection forestland, the highest number of stems removed were due to natural death followed by poles, firewood collection and timber (Table 5). In terms of AGC, the highest AGC were 
removed as timber followed by charcoal, natural death, poles and fire. Grazing by domestic animals, carvings and grazing by wild animals accounted for the least AGC removals in protection forest. In other land use types such as production forest, grazing land, shifting cultivation, water bodies or swamps and wildlife reserves, drivers of removals appear to be changing leading positions in terms of the number of stems and AGC.

Table 1

Drivers and their corresponding number of stems and AGC removals in mainland Tanzania

\begin{tabular}{|c|c|c|c|c|c|}
\hline Drivers & Stemsha $a^{-1} \mathrm{yr}^{-1}$ & Stem \% & AGBtha ${ }^{-1} \mathrm{yr}^{-1}$ & AGCtCha $^{-1} \mathrm{yr}^{-1}$ & Agb\% \\
\hline Timber & 0.780 & 7.000 & 0.244 & 0.119 & 20.173 \\
\hline Fire & 0.845 & 7.581 & 0.196 & 0.096 & 16.235 \\
\hline Shifting cultivation & 2.741 & 24.595 & 0.191 & 0.093 & 15.788 \\
\hline Charcoal & 1.747 & 15.672 & 0.182 & 0.089 & 15.085 \\
\hline Natural death & 1.233 & 11.061 & 0.160 & 0.079 & 13.268 \\
\hline Firewood collection & 1.376 & 12.343 & 0.089 & 0.043 & 7.331 \\
\hline Poles & 1.588 & 14.250 & 0.078 & 0.038 & 6.494 \\
\hline Unknown & 0.472 & 4.233 & 0.047 & 0.023 & 3.871 \\
\hline Grazing wild & 0.256 & 2.294 & 0.014 & 0.007 & 1.192 \\
\hline Carvings & 0.050 & 0.450 & 0.004 & 0.002 & 0.350 \\
\hline Grazing domestic & 0.053 & 0.471 & 0.002 & 0.001 & 0.203 \\
\hline Mining & 0.006 & 0.051 & 0.000 & 0.000 & 0.010 \\
\hline
\end{tabular}


Table 2

Drivers and their corresponding number of stems and AGC removals in zones of mainland Tanzania

\begin{tabular}{|c|c|c|c|c|c|c|}
\hline $\begin{array}{l}\text { Zone } \\
\text { names }\end{array}$ & Drivers & Stems/ha/yr & Stem\% & Agbt/ha/yr & AgctC/ha/yr & AgctC\% \\
\hline \multirow[t]{10}{*}{ Central } & Charcoal & 3.687 & 28.472 & 0.187 & 0.092 & 49.202 \\
\hline & $\begin{array}{l}\text { Firewood } \\
\text { collection }\end{array}$ & 3.653 & 28.213 & 0.055 & 0.027 & 14.429 \\
\hline & Timber & 1.334 & 10.303 & 0.046 & 0.023 & 12.215 \\
\hline & $\begin{array}{l}\text { Shifting } \\
\text { cultivation }\end{array}$ & 1.808 & 13.961 & 0.042 & 0.020 & 10.969 \\
\hline & Natural death & 0.693 & 5.351 & 0.027 & 0.013 & 7.131 \\
\hline & Fire & 0.870 & 6.722 & 0.018 & 0.009 & 4.662 \\
\hline & Poles & 0.820 & 6.332 & 0.003 & 0.001 & 0.787 \\
\hline & Grazing domestic & 0.019 & 0.148 & 0.001 & 0.001 & 0.380 \\
\hline & Grazing wild & 0.065 & 0.498 & 0.001 & 0.000 & 0.225 \\
\hline & Total & 12.949 & 100 & 0.380 & 0.186 & 100 \\
\hline \multirow[t]{11}{*}{ Eastern } & Timber & 5.812 & 12.466 & 0.746 & 0.366 & 30.732 \\
\hline & Charcoal & 11.014 & 23.625 & 0.655 & 0.321 & 26.979 \\
\hline & Natural death & 7.904 & 16.954 & 0.405 & 0.198 & 16.670 \\
\hline & $\begin{array}{l}\text { Shifting } \\
\text { cultivation }\end{array}$ & 5.151 & 11.050 & 0.189 & 0.092 & 7.772 \\
\hline & $\begin{array}{l}\text { Firewood } \\
\text { collection }\end{array}$ & 8.157 & 17.497 & 0.178 & 0.087 & 7.337 \\
\hline & Poles & 5.137 & 11.019 & 0.097 & 0.048 & 4.004 \\
\hline & Fire & 1.022 & 2.193 & 0.079 & 0.039 & 3.258 \\
\hline & Grazing wild & 2.201 & 4.722 & 0.069 & 0.034 & 2.844 \\
\hline & Carvings & 0.199 & 0.426 & 0.010 & 0.005 & 0.396 \\
\hline & Grazing domestic & 0.023 & 0.048 & 0.000 & 0.000 & 0.008 \\
\hline & Total & 46.62 & 100 & 2.428 & 1.19 & 100 \\
\hline \multirow[t]{3}{*}{ Lake } & Fire & 0.515 & 6.604 & 0.089 & 0.044 & 33.115 \\
\hline & Poles & 2.303 & 29.520 & 0.049 & 0.024 & 18.096 \\
\hline & $\begin{array}{l}\text { Firewood } \\
\text { collection }\end{array}$ & 2.555 & 32.755 & 0.042 & 0.021 & 15.674 \\
\hline
\end{tabular}




\begin{tabular}{|lllllll|}
\hline $\begin{array}{l}\text { Zone } \\
\text { names }\end{array}$ & Drivers & Stems/ha/yr & Stem\% & Agbt/ha/yr & AgctC/ha/yr & AgctC\% \\
\hline & Timber & 0.379 & 4.862 & 0.035 & 0.017 & 13.031 \\
\hline & Charcoal & 0.663 & 8.495 & 0.023 & 0.011 & 8.555 \\
\hline Natural death & 0.782 & 10.023 & 0.023 & 0.011 & 8.720 \\
\hline $\begin{array}{l}\text { Shifting } \\
\text { cultivation }\end{array}$ & 0.417 & 5.347 & 0.005 & 0.003 & 1.901 \\
\hline Grazing wild & 0.187 & 2.394 & 0.002 & 0.001 & 0.909 \\
\hline Total & 7.801 & 100 & 0.268 & 0.132 & 100 \\
\hline Charcoal & 8.040 & 21.454 & 0.331 & 0.162 & 22.579 \\
\hline $\begin{array}{l}\text { Shifting } \\
\text { cultivation }\end{array}$ & 13.459 & 35.915 & 0.324 & 0.159 & 22.145 \\
\hline Timber & 1.257 & 3.355 & 0.301 & 0.147 & 20.525 \\
\hline Poles & 6.117 & 16.325 & 0.209 & 0.102 & 14.248 \\
\hline Natural death & 4.459 & 11.898 & 0.166 & 0.081 & 11.301 \\
\hline $\begin{array}{l}\text { Firewood } \\
\text { collection }\end{array}$ & 2.881 & 7.688 & 0.103 & 0.05 & 7.006 \\
\hline Fire & 1.092 & 2.915 & 0.030 & 0.015 & 2.072 \\
\hline Grazing wild & 0.168 & 0.449 & 0.002 & 0.001 & 0.124 \\
\hline Total & 37.473 & 100 & 1.466 & 0.717 & 100 \\
\hline
\end{tabular}


Table 2

cont....

\begin{tabular}{|c|c|c|c|c|c|c|}
\hline \multirow[t]{9}{*}{ Southern highlands } & Natural death & 2.397 & 25.501 & 0.332 & 0.163 & 59.949 \\
\hline & Poles & 3.026 & 32.2 & 0.08 & 0.039 & 14.403 \\
\hline & Timber & 0.649 & 6.908 & 0.075 & 0.037 & 13.576 \\
\hline & Firewood collection & 2.294 & 24.407 & 0.052 & 0.026 & 9.44 \\
\hline & Shifting cultivation & 0.621 & 6.61 & 0.008 & 0.004 & 1.401 \\
\hline & Grazing domestic & 0.091 & 0.964 & 0.004 & 0.002 & 0.731 \\
\hline & Charcoal & 0.258 & 2.741 & 0.002 & 0.001 & 0.421 \\
\hline & Grazing wild & 0.063 & 0.67 & 0 & 0 & 0.079 \\
\hline & Total & 9.399 & 100 & 0.553 & 0.272 & 100 \\
\hline \multirow[t]{12}{*}{ Southern } & Fire & 4.143 & 14.368 & 0.586 & 0.287 & 36.115 \\
\hline & Timber & 2.149 & 7.455 & 0.304 & 0.149 & 18.729 \\
\hline & Shifting cultivation & 7.009 & 24.309 & 0.293 & 0.144 & 18.049 \\
\hline & Natural death & 7.122 & 24.7 & 0.183 & 0.09 & 11.257 \\
\hline & Poles & 4.477 & 15.528 & 0.112 & 0.055 & 6.923 \\
\hline & Charcoal & 0.885 & 3.07 & 0.064 & 0.031 & 3.916 \\
\hline & Firewood collection & 1.54 & 5.342 & 0.048 & 0.024 & 2.961 \\
\hline & Grazing wild & 0.884 & 3.067 & 0.019 & 0.01 & 1.199 \\
\hline & Carvings & 0.311 & 1.078 & 0.011 & 0.006 & 0.702 \\
\hline & Grazing domestic & 0.233 & 0.809 & 0.002 & 0.001 & 0.121 \\
\hline & Mining & 0.079 & 0.273 & 0 & 0 & 0.028 \\
\hline & Total & 28.832 & 100 & 1.622 & 0.797 & 100 \\
\hline \multirow[t]{7}{*}{ Western } & Timber & 1.782 & 11.582 & 0.163 & 0.08 & 27.946 \\
\hline & Shifting cultivation & 5.317 & 34.564 & 0.127 & 0.062 & 21.766 \\
\hline & Natural death & 1.592 & 10.348 & 0.073 & 0.036 & 12.584 \\
\hline & Firewood collection & 1.866 & 12.128 & 0.065 & 0.032 & 11.195 \\
\hline & Charcoal & 1.364 & 8.866 & 0.059 & 0.029 & 10.053 \\
\hline & Fire & 0.922 & 5.993 & 0.059 & 0.029 & 10.169 \\
\hline & Poles & 2.419 & 15.726 & 0.031 & 0.015 & 5.366 \\
\hline
\end{tabular}




\begin{tabular}{|lllllll|}
\hline Southern highlands & Natural death & $\mathbf{2 . 3 9 7}$ & $\mathbf{2 5 . 5 0 1}$ & $\mathbf{0 . 3 3 2}$ & $\mathbf{0 . 1 6 3}$ & $\mathbf{5 9 . 9 4 9}$ \\
& Grazing domestic & 0.025 & 0.163 & 0.005 & 0.002 & 0.794 \\
& Grazing wild & 0.097 & 0.632 & 0.001 & 0 & 0.128 \\
Total & 15.384 & 100 & $\mathbf{0 . 5 8 3}$ & $\mathbf{0 . 2 8 5}$ & $\mathbf{1 0 0}$ \\
\hline
\end{tabular}


Table 3

Drivers and their corresponding number of stems and AGC removals in miombo woodlands vegetation subtypes of mainland Tanzania

\begin{tabular}{|c|c|c|c|c|c|c|}
\hline $\begin{array}{l}\text { Vegetation } \\
\text { types }\end{array}$ & Drivers & Stems/ha/yr & $\begin{array}{l}\text { Stems } \\
\%\end{array}$ & Agbt/ha/yr & AgctC/ha/yr & Agct/ha/yr\% \\
\hline \multirow{12}{*}{$\begin{array}{l}\text { Closed } \\
\text { woodlands } \\
\text { (crown cover } \\
>40 \% \text { ) }\end{array}$} & $\begin{array}{l}\text { Natural } \\
\text { death }\end{array}$ & 2.070 & 28.528 & 0.251 & 0.123 & 26.309 \\
\hline & Timber & 0.847 & 11.680 & 0.250 & 0.122 & 26.236 \\
\hline & $\begin{array}{l}\text { Shifting } \\
\text { cultivation }\end{array}$ & 0.782 & 10.782 & 0.183 & 0.090 & 19.174 \\
\hline & Unknown & 0.384 & 5.297 & 0.069 & 0.034 & 7.243 \\
\hline & Fire & 0.642 & 8.843 & 0.063 & 0.031 & 6.657 \\
\hline & Poles & 1.041 & 14.350 & 0.044 & 0.022 & 4.662 \\
\hline & $\begin{array}{l}\text { Firewood } \\
\text { collection }\end{array}$ & 0.675 & 9.297 & 0.040 & 0.020 & 4.219 \\
\hline & Charcoal & 0.414 & 5.707 & 0.040 & 0.020 & 4.192 \\
\hline & $\begin{array}{l}\text { Grazing } \\
\text { wild }\end{array}$ & 0.338 & 4.658 & 0.010 & 0.005 & 1.091 \\
\hline & Carvings & 0.027 & 0.366 & 0.001 & 0.001 & 0.122 \\
\hline & $\begin{array}{l}\text { Grazing } \\
\text { domestic }\end{array}$ & 0.036 & 0.492 & 0.001 & 0.000 & 0.094 \\
\hline & Total & 7.255 & 100 & 0.953 & 0.467 & 100 \\
\hline \multirow{7}{*}{$\begin{array}{l}\text { Open } \\
\text { woodlands } \\
\text { (Crown cover } \\
\text { between 10- } \\
40 \% \text { ) }\end{array}$} & Timber & 11.005 & 51.300 & 0.262 & 0.128 & 20.443 \\
\hline & Fire & 0.903 & 4.210 & 0.246 & 0.121 & 19.225 \\
\hline & Charcoal & 1.454 & 6.779 & 0.193 & 0.095 & 15.070 \\
\hline & $\begin{array}{l}\text { Natural } \\
\text { death }\end{array}$ & 1.686 & 7.860 & 0.167 & 0.082 & 13.061 \\
\hline & $\begin{array}{l}\text { Shifting } \\
\text { cultivation }\end{array}$ & 2.448 & 11.409 & 0.158 & 0.078 & 12.360 \\
\hline & $\begin{array}{l}\text { Firewood } \\
\text { collection }\end{array}$ & 1.550 & 7.226 & 0.101 & 0.049 & 7.882 \\
\hline & Poles & 1.538 & 7.168 & 0.086 & 0.042 & 6.702 \\
\hline
\end{tabular}




\begin{tabular}{|c|c|c|c|c|c|c|}
\hline $\begin{array}{l}\text { Vegetation } \\
\text { types }\end{array}$ & Drivers & Stems/ha/yr & $\begin{array}{l}\text { Stems } \\
\%\end{array}$ & Agbt/ha/yr & AgctC/ha/yr & Agct/ha/yr\% \\
\hline & Unknown & 0.499 & 2.328 & 0.042 & 0.021 & 3.297 \\
\hline & $\begin{array}{l}\text { Grazing } \\
\text { wild }\end{array}$ & 0.245 & 1.142 & 0.017 & 0.008 & 1.288 \\
\hline & Carvings & 0.055 & 0.258 & 0.005 & 0.003 & 0.419 \\
\hline & $\begin{array}{l}\text { Grazing } \\
\text { domestic }\end{array}$ & 0.061 & 0.284 & 0.003 & 0.002 & 0.241 \\
\hline & Mining & 0.008 & 0.037 & 0.000 & 0.000 & 0.013 \\
\hline & Total & 21.453 & 100 & 1.282 & 0.628 & 100 \\
\hline \multirow{11}{*}{$\begin{array}{l}\text { Woodlands } \\
\text { with scattered } \\
\text { cropland }\end{array}$} & $\begin{array}{l}\text { Shifting } \\
\text { cultivation }\end{array}$ & 16.587 & 58.998 & 0.735 & 0.360 & 47.938 \\
\hline & Charcoal & 1.751 & 6.228 & 0.241 & 0.118 & 15.713 \\
\hline & Timber & 0.382 & 1.360 & 0.180 & 0.088 & 11.715 \\
\hline & $\begin{array}{l}\text { Firewood } \\
\text { collection }\end{array}$ & 2.032 & 7.228 & 0.127 & 0.062 & 8.259 \\
\hline & Poles & 4.994 & 17.764 & 0.121 & 0.059 & 7.914 \\
\hline & $\begin{array}{l}\text { Natural } \\
\text { death }\end{array}$ & 0.858 & 3.051 & 0.073 & 0.036 & 4.759 \\
\hline & Fire & 0.912 & 3.244 & 0.039 & 0.019 & 2.541 \\
\hline & Unknown & 0.452 & 1.606 & 0.011 & 0.005 & 0.700 \\
\hline & Pole & 0.063 & 0.225 & 0.006 & 0.003 & 0.383 \\
\hline & Carvings & 0.083 & 0.297 & 0.001 & 0.001 & 0.080 \\
\hline & Total & 28.114 & 100 & 1.533 & 0.751 & 100 \\
\hline
\end{tabular}


Table 4

Drivers and their corresponding number of stems and AGC removals in ownership types of miombo woodlands in Mainland Tanzania

\begin{tabular}{|c|c|c|c|c|c|c|}
\hline $\begin{array}{l}\text { Ownership } \\
\text { types }\end{array}$ & Drivers & Stems/ha/yr & $\begin{array}{l}\text { Stems } \\
\%\end{array}$ & Agbt/ha/yr & AgctC/ha/yr & $\begin{array}{l}\text { AgctC } \\
\%\end{array}$ \\
\hline \multirow{11}{*}{$\begin{array}{l}\text { Central } \\
\text { Government }\end{array}$} & Timber & 0.507 & 10.511 & 0.114 & 0.056 & 26.306 \\
\hline & Natural death & 1.503 & 31.143 & 0.103 & 0.050 & 23.651 \\
\hline & Fire & 0.695 & 14.409 & 0.079 & 0.039 & 18.124 \\
\hline & Charcoal & 0.284 & 5.888 & 0.037 & 0.018 & 8.604 \\
\hline & Grazing wild & 0.561 & 11.617 & 0.037 & 0.018 & 8.467 \\
\hline & $\begin{array}{l}\text { Firewood } \\
\text { collection }\end{array}$ & 0.331 & 6.851 & 0.026 & 0.013 & 6.040 \\
\hline & Poles & 0.551 & 11.406 & 0.020 & 0.010 & 4.652 \\
\hline & $\begin{array}{l}\text { Shifting } \\
\text { cultivation }\end{array}$ & 0.372 & 7.704 & 0.016 & 0.008 & 3.792 \\
\hline & Carvings & 0.011 & 0.218 & 0.001 & 0.000 & 0.232 \\
\hline & $\begin{array}{l}\text { Grazing } \\
\text { domestic }\end{array}$ & 0.012 & 0.254 & 0.001 & 0.000 & 0.132 \\
\hline & Total & 4.827 & 100 & 0.434 & 0.213 & 100 \\
\hline \multirow{10}{*}{$\begin{array}{l}\text { Local } \\
\text { Government }\end{array}$} & Natural death & 1.684 & 23.388 & 0.310 & 0.152 & 33.885 \\
\hline & Charcoal & 1.738 & 24.134 & 0.217 & 0.106 & 23.743 \\
\hline & Timber & 0.988 & 13.719 & 0.210 & 0.103 & 23.032 \\
\hline & $\begin{array}{l}\text { Firewood } \\
\text { collection }\end{array}$ & 2.315 & 32.141 & 0.140 & 0.068 & 15.288 \\
\hline & Fire & 0.225 & 3.128 & 0.031 & 0.015 & 3.446 \\
\hline & $\begin{array}{l}\text { Shifting } \\
\text { cultivation }\end{array}$ & 0.118 & 1.634 & 0.004 & 0.002 & 0.471 \\
\hline & Poles & 0.106 & 1.476 & 0.001 & 0.000 & 0.080 \\
\hline & $\begin{array}{l}\text { Grazing } \\
\text { domestic }\end{array}$ & 0.019 & 0.269 & 0.000 & 0.000 & 0.054 \\
\hline & Grazing wild & 0.008 & 0.111 & 0.000 & 0.000 & 0.002 \\
\hline & Total & 7.201 & 100 & 0.914 & 0.448 & 100 \\
\hline Village land & Fire & 1.000 & 7.911 & 0.315 & 0.154 & 22.305 \\
\hline
\end{tabular}




\begin{tabular}{|lllllll|}
\hline $\begin{array}{l}\text { Ownership } \\
\text { types }\end{array}$ & Drivers & Stems/ha/yr & $\begin{array}{l}\text { Stems } \\
\%\end{array}$ & Agbt/ha/yr & AgctC/ha/yr & $\begin{array}{l}\text { AgctC } \\
\%\end{array}$ \\
\hline Timber & 0.841 & 6.654 & 0.278 & 0.136 & 19.694 \\
\hline Natural death & 2.000 & 15.815 & 0.227 & 0.111 & 16.082 \\
\hline Charcoal & 1.363 & 10.779 & 0.177 & 0.087 & 12.550 \\
\hline $\begin{array}{l}\text { Shifting } \\
\text { cultivation }\end{array}$ & 3.038 & 24.024 & 0.160 & 0.079 & 11.366 \\
\hline Poles & 2.337 & 18.479 & 0.122 & 0.060 & 8.678 \\
\hline $\begin{array}{l}\text { Firewood } \\
\text { collection }\end{array}$ & 1.755 & 13.882 & 0.115 & 0.056 & 8.156 \\
\hline Grazing wild & 0.170 & 1.347 & 0.008 & 0.004 & 0.564 \\
\hline Carvings & 0.066 & 0.522 & 0.004 & 0.002 & 0.300 \\
\hline $\begin{array}{l}\text { Grazing } \\
\text { domestic }\end{array}$ & 0.063 & 0.499 & 0.004 & 0.002 & 0.288 \\
\hline Mining & 0.011 & 0.087 & 0.000 & 0.000 & 0.017 \\
\hline Total & 12.645 & 100 & 1.411 & 0.691 & 100 \\
\hline
\end{tabular}


Table 4

cont....

\begin{tabular}{|c|c|c|c|c|c|c|}
\hline \multirow[t]{10}{*}{ Private Land } & Shifting cultivation & 11.128 & 59.113 & 1.102 & 0.540 & 60.139 \\
\hline & Charcoal & 2.466 & 13.101 & 0.355 & 0.174 & 19.388 \\
\hline & Timber & 0.625 & 3.320 & 0.135 & 0.066 & 7.344 \\
\hline & Firewood collection & 1.913 & 10.160 & 0.116 & 0.057 & 6.348 \\
\hline & Poles & 1.991 & 10.577 & 0.059 & 0.029 & 3.216 \\
\hline & Natural death & 0.374 & 1.987 & 0.046 & 0.023 & 2.517 \\
\hline & Fire & 0.278 & 1.478 & 0.016 & 0.008 & 0.872 \\
\hline & Pole & 0.033 & 0.175 & 0.003 & 0.001 & 0.167 \\
\hline & Grazing wild & 0.016 & 0.088 & 0.000 & 0.000 & 0.009 \\
\hline & Total & 18.825 & 100 & 1.832 & 0.898 & 100 \\
\hline \multirow[t]{11}{*}{ General land } & Timber & 1.577 & 13.068 & 0.807 & 0.395 & 48.146 \\
\hline & Natural death & 2.719 & 22.537 & 0.247 & 0.121 & 14.754 \\
\hline & Charcoal & 2.163 & 17.925 & 0.226 & 0.111 & 13.470 \\
\hline & Fire & 1.911 & 15.838 & 0.155 & 0.076 & 9.268 \\
\hline & Shifting cultivation & 1.308 & 10.839 & 0.086 & 0.042 & 5.127 \\
\hline & Poles & 0.854 & 7.080 & 0.085 & 0.042 & 5.059 \\
\hline & Carvings & 0.256 & 2.124 & 0.034 & 0.017 & 2.056 \\
\hline & Firewood collection & 0.721 & 5.976 & 0.027 & 0.013 & 1.586 \\
\hline & Grazing wild & 0.260 & 2.153 & 0.006 & 0.003 & 0.344 \\
\hline & Grazing domestic & 0.297 & 2.461 & 0.003 & 0.002 & 0.191 \\
\hline & Total & 12.065 & 100 & 1.675 & 0.821 & 100 \\
\hline \multirow[t]{3}{*}{ Unknown } & Firewood collection & 1.051 & 68.394 & 0.010 & 0.005 & 69.823 \\
\hline & Natural death & 0.486 & 31.606 & 0.004 & 0.002 & 30.177 \\
\hline & Total & 1.536 & 100 & 0.014 & 0.007 & 100 \\
\hline
\end{tabular}


Table 5

Drivers and their corresponding number of stems and biomass removals in land use types of miombo woodlands in mainland Tanzania

\begin{tabular}{|c|c|c|c|c|c|c|}
\hline $\begin{array}{l}\text { Ownership } \\
\text { types }\end{array}$ & Drivers & Stems/ha/yr & $\begin{array}{l}\text { Stems } \\
\%\end{array}$ & Agbt/ha/yr & AgctC/ha/yr & $\begin{array}{l}\text { AgctC } \\
\%\end{array}$ \\
\hline \multirow{13}{*}{$\begin{array}{l}\text { Production } \\
\text { forest }\end{array}$} & Fire & 1.144 & 10.354 & 0.390 & 0.191 & 26.726 \\
\hline & Timber & 1.086 & 9.831 & 0.380 & 0.186 & 26.061 \\
\hline & Charcoal & 1.426 & 12.903 & 0.203 & 0.099 & 13.925 \\
\hline & Natural death & 2.294 & 20.764 & 0.167 & 0.082 & 11.473 \\
\hline & Poles & 1.968 & 17.815 & 0.108 & 0.053 & 7.385 \\
\hline & $\begin{array}{l}\text { Firewood } \\
\text { collection }\end{array}$ & 1.582 & 14.318 & 0.106 & 0.052 & 7.284 \\
\hline & $\begin{array}{l}\text { Shifting } \\
\text { cultivation }\end{array}$ & 0.668 & 6.046 & 0.048 & 0.024 & 3.313 \\
\hline & Unknown & 0.450 & 4.077 & 0.031 & 0.015 & 2.104 \\
\hline & Grazing wild & 0.230 & 2.082 & 0.010 & 0.005 & 0.717 \\
\hline & Carvings & 0.097 & 0.874 & 0.010 & 0.005 & 0.653 \\
\hline & $\begin{array}{l}\text { Grazing } \\
\text { domestic }\end{array}$ & 0.089 & 0.810 & 0.005 & 0.002 & 0.339 \\
\hline & Mining & 0.014 & 0.127 & 0.000 & 0.000 & 0.020 \\
\hline & Total & 11.049 & 100 & 1.458 & 0.714 & 100 \\
\hline \multirow{9}{*}{$\begin{array}{l}\text { Protection } \\
\text { forest }\end{array}$} & Natural death & 3.749 & 40.101 & 0.176 & 0.086 & 44.792 \\
\hline & Timber & 0.936 & 10.012 & 0.090 & 0.044 & 22.917 \\
\hline & Fire & 1.754 & 18.761 & 0.069 & 0.029 & 15.104 \\
\hline & Grazing wild & 1.567 & 16.761 & 0.045 & 0.022 & 11.458 \\
\hline & Poles & 0.457 & 4.888 & 0.010 & 0.005 & 2.604 \\
\hline & $\begin{array}{l}\text { Firewood } \\
\text { collection }\end{array}$ & 0.671 & 7.177 & 0.008 & 0.004 & 2.083 \\
\hline & Charcoal & 0.083 & 0.888 & 0.004 & 0.002 & 1.042 \\
\hline & Unknown & 0.052 & 0.556 & 0.002 & 0.000 & 0.000 \\
\hline & $\begin{array}{l}\text { Grazing } \\
\text { domestic }\end{array}$ & 0.061 & 0.652 & 0.001 & 0.000 & 0.000 \\
\hline
\end{tabular}




\begin{tabular}{|c|c|c|c|c|c|c|}
\hline $\begin{array}{l}\text { Ownership } \\
\text { types }\end{array}$ & Drivers & Stems/ha/yr & $\begin{array}{l}\text { Stems } \\
\%\end{array}$ & Agbt/ha/yr & AgctC/ha/yr & $\begin{array}{l}\text { AgctC } \\
\%\end{array}$ \\
\hline & $\begin{array}{l}\text { Shifting } \\
\text { cultivation }\end{array}$ & 0.019 & 0.203 & 0.000 & 0.000 & 0.000 \\
\hline & Total & 9.349 & 100 & 0.405 & 0.192 & 100 \\
\hline \multirow[t]{11}{*}{ Wildlife reserve } & Natural death & 1.985 & 38.544 & 0.213 & 0.104 & 40.532 \\
\hline & Timber & 0.364 & 7.079 & 0.121 & 0.059 & 23.125 \\
\hline & Fire & 0.903 & 17.534 & 0.079 & 0.039 & 15.010 \\
\hline & Grazing wild & 0.932 & 18.091 & 0.060 & 0.029 & 11.398 \\
\hline & Unknown & 0.282 & 5.473 & 0.021 & 0.010 & 4.080 \\
\hline & Poles & 0.184 & 3.572 & 0.014 & 0.007 & 2.605 \\
\hline & $\begin{array}{l}\text { Firewood } \\
\text { collection }\end{array}$ & 0.367 & 7.131 & 0.010 & 0.005 & 1.992 \\
\hline & Charcoal & 0.069 & 1.343 & 0.005 & 0.003 & 0.994 \\
\hline & $\begin{array}{l}\text { Grazing } \\
\text { domestic }\end{array}$ & 0.052 & 1.005 & 0.001 & 0.001 & 0.235 \\
\hline & $\begin{array}{l}\text { Shifting } \\
\text { cultivation }\end{array}$ & 0.012 & 0.229 & 0.000 & 0.001 & 0.030 \\
\hline & Total & 5.149 & 100 & 0.525 & 0.257 & 100 \\
\hline \multirow[t]{10}{*}{$\begin{array}{l}\text { Shifting } \\
\text { cultivation }\end{array}$} & $\begin{array}{l}\text { Shifting } \\
\text { cultivation }\end{array}$ & 21.885 & 61.492 & 1.664 & 0.815 & 61.559 \\
\hline & Timber & 0.985 & 2.768 & 0.310 & 0.152 & 11.486 \\
\hline & Charcoal & 2.368 & 6.652 & 0.187 & 0.092 & 6.930 \\
\hline & $\begin{array}{l}\text { Firewood } \\
\text { collection }\end{array}$ & 2.463 & 6.920 & 0.150 & 0.073 & 5.543 \\
\hline & Poles & 4.520 & 12.700 & 0.134 & 0.066 & 4.973 \\
\hline & Unknown & 1.471 & 4.133 & 0.118 & 0.058 & 4.382 \\
\hline & Natural death & 0.952 & 2.675 & 0.094 & 0.046 & 3.462 \\
\hline & Fire & 0.870 & 2.445 & 0.044 & 0.022 & 1.630 \\
\hline & Carvings & 0.077 & 0.216 & 0.001 & 0.000 & 0.035 \\
\hline & Total & 35.591 & 100 & 2.703 & 1.324 & 100 \\
\hline Agriculture & $\begin{array}{l}\text { Shifting } \\
\text { cultivation }\end{array}$ & 12.914 & 49.747 & 0.884 & 0.433 & 46.721 \\
\hline
\end{tabular}




\begin{tabular}{|c|c|c|c|c|c|c|}
\hline \multirow[t]{12}{*}{$\begin{array}{l}\text { Ownership } \\
\text { types }\end{array}$} & Drivers & Stems/ha/yr & $\begin{array}{l}\text { Stems } \\
\%\end{array}$ & Agbt/ha/yr & AgctC/ha/yr & $\begin{array}{l}\text { AgctC } \\
\%\end{array}$ \\
\hline & $\begin{array}{l}\text { Firewood } \\
\text { collection }\end{array}$ & 4.776 & 18.399 & 0.411 & 0.201 & 21.685 \\
\hline & Charcoal & 2.107 & 8.116 & 0.217 & 0.107 & 11.484 \\
\hline & Unknown & 1.173 & 4.519 & 0.105 & 0.051 & 5.547 \\
\hline & Fire & 0.807 & 3.107 & 0.093 & 0.046 & 4.932 \\
\hline & Timber & 0.405 & 1.559 & 0.072 & 0.035 & 3.817 \\
\hline & Poles & 2.669 & 10.282 & 0.065 & 0.032 & 3.447 \\
\hline & Natural death & 0.962 & 3.706 & 0.036 & 0.018 & 1.906 \\
\hline & Pole & 0.068 & 0.261 & 0.006 & 0.003 & 0.332 \\
\hline & $\begin{array}{l}\text { Grazing } \\
\text { domestic }\end{array}$ & 0.034 & 0.130 & 0.002 & 0.001 & 0.101 \\
\hline & Grazing wild & 0.045 & 0.174 & 0.001 & 0.001 & 0.027 \\
\hline & Total & 25.959 & 100 & 1.893 & 0.928 & 100 \\
\hline \multirow[t]{12}{*}{ Grazing land } & $\begin{array}{l}\text { Grazing } \\
\text { domestic }\end{array}$ & 1.830 & 17.790 & 0.606 & 0.297 & 43.763 \\
\hline & Charcoal & 2.339 & 22.742 & 0.410 & 0.201 & 29.570 \\
\hline & $\begin{array}{l}\text { Shifting } \\
\text { cultivation }\end{array}$ & 2.754 & 26.783 & 0.101 & 0.049 & 7.280 \\
\hline & Poles & 1.405 & 13.657 & 0.080 & 0.039 & 5.767 \\
\hline & $\begin{array}{l}\text { Firewood } \\
\text { collection }\end{array}$ & 1.281 & 12.454 & 0.078 & 0.038 & 5.652 \\
\hline & Fire & 0.297 & 2.888 & 0.055 & 0.027 & 3.944 \\
\hline & Timber & 0.153 & 1.492 & 0.037 & 0.018 & 2.669 \\
\hline & Unknown & 0.171 & 1.667 & 0.014 & 0.007 & 0.985 \\
\hline & Carvings & 0.017 & 0.162 & 0.003 & 0.001 & 0.201 \\
\hline & Natural death & 0.022 & 0.216 & 0.002 & 0.001 & 0.121 \\
\hline & Grazing wild & 0.015 & 0.149 & 0.001 & 0.001 & 0.049 \\
\hline & Total & 10.284 & 100 & 1.385 & 0.679 & 100 \\
\hline Built up area & $\begin{array}{l}\text { Firewood } \\
\text { collection }\end{array}$ & 7.798 & 100 & 0.319 & 0.156 & 100 \\
\hline
\end{tabular}




\begin{tabular}{|c|c|c|c|c|c|c|}
\hline $\begin{array}{l}\text { Ownership } \\
\text { types }\end{array}$ & Drivers & Stems/ha/yr & $\begin{array}{l}\text { Stems } \\
\%\end{array}$ & Agbt/ha/yr & AgctC/ha/yr & $\begin{array}{l}\text { AgctC } \\
\%\end{array}$ \\
\hline & Total & 7.798 & 100 & 0.319 & 0.156 & 100 \\
\hline \multirow{3}{*}{$\begin{array}{l}\text { Water } \\
\text { body/swamp }\end{array}$} & Timber & 12.758 & 66.667 & 2.599 & 1.273 & 95.994 \\
\hline & Poles & 6.379 & 33.333 & 0.108 & 0.053 & 4.006 \\
\hline & Total & 19.137 & 100 & 2.707 & 1.327 & 100 \\
\hline \multirow[t]{8}{*}{ Other land } & Fire & 1.371 & 27.861 & 0.085 & 0.042 & 35.878 \\
\hline & Poles & 0.415 & 8.435 & 0.045 & 0.022 & 19.128 \\
\hline & $\begin{array}{l}\text { Firewood } \\
\text { collection }\end{array}$ & 0.605 & 12.289 & 0.041 & 0.020 & 17.176 \\
\hline & Natural death & 1.102 & 22.398 & 0.033 & 0.016 & 13.793 \\
\hline & Charcoal & 1.105 & 22.464 & 0.031 & 0.015 & 13.159 \\
\hline & Grazing wild & 0.138 & 2.808 & 0.001 & 0.001 & 0.558 \\
\hline & $\begin{array}{l}\text { Shifting } \\
\text { cultivation }\end{array}$ & 0.184 & 3.744 & 0.001 & 0.001 & 0.309 \\
\hline & Total & 4.920 & 100 & 0.238 & 0.116 & 100 \\
\hline
\end{tabular}

\section{Discussion}

The overall objective of this paper was to identify the drivers of AGC removals and to quantify the contributions of each driver to the variation of AGC removals and hence carbon emissions in miombo woodlands in Tanzania using NAFORMA data set. In this study, drivers and their corresponding estimates of $A G C$ and number of stems removals have been reported. The carbon stored in the aboveground biomass (AGB) pool is typically the largest among the Intergovernmental Panel for Climate Change (IPCC) carbon pools for REDD + reporting purposes. It is understood that while removals by shifting cultivation fire, firewood collection and charcoal, results immediately into carbon emissions, it is not the case with removals for timber, carvings and poles which may end up in construction and furniture whose emissions may be delayed. Nonetheless, timber in the form of furniture, carvings or construction is more in the process of contributing to emissions although delayed. Due to the uncertainty of time taken for timber to act as stored carbon all removals are assumed to eventually to contribute to emissions.

\section{Drivers of aboveground carbon and number of stems removals}

Several drivers contributed number of stems and AGC removals in mainland Tanzania. These drivers included charcoal, wildfire, firewood collection, grazing by both wildlife and domesticated animals, 
carving, poles, shifting cultivation, timber, and mining activities. Since drivers of AGC removals are similar to drivers for forest degradation in the woodlands meant for both protective and production purposes, comparison across studies were based on studies conducted to determine forest degradation drivers. The result found in the present study is comparable to results found in miombo woodland in Masito forest in western Tanzania and Liwale district southern Tanzania [23, 24, 25]. These studies documented only six drivers responsible for forest degradation. Sites specific and the methodologies applied on these studies explains fewer documentation of drivers. On the other hand, [26] documented ten drivers for forest degradation in Philippines that agrees with results from the present study. The methodology employed, particularly on the sampling procedures could explain the similarity.

In terms of the contribution of drivers on the number of stems and AGC removals nationally, removals by shifting cultivation, natural death, poles and charcoal production account for the highest number of stem removals. The reason could be attributed by high demand of charcoal in the country for cooking energy in which small diameter trees are involved. Tanzania's annual consumption of charcoal is $1,658,000$ tons [27]. About $85 \%$ of the total urban population depends on charcoal for household cooking and energy for small and medium enterprises [28]. Additionally, more than $40 \%$ of the tree removals can be attributed to charcoal use alone in Tanzania [29]. Higher removals by shifting cultivation is probably due to intensification of shifting cultivation in Tanzania. Shifting cultivation in Tanzania occupies $7.6 \%$ of the total country land area and 33\% of area classified as woodlands in Tanzania [22]. Other scholars [30, 31] asserted that shifting cultivation contribute more to forest degradation due rising demand for agricultural products, dietary changes, agricultural trade and adjustment. Firewood collection and poles on the other hand, rank third and fourth in taking large amount of stems in the woodlands. This is probably because; firewood is the main source of energy rural areas [32]. The same author noted that, lack of alternative and affordable sources of energy dependence of communities on forests. Construction purposes both in the rural and urban areas probably account for higher removals of trees as poles. Furthermore, climate change impacts like diseases eruptions and severe drought naturally kills trees. These effects have recently increased tremendously. Mining and grazing by domesticated animals appeared as the least drivers responsible for stems removals. This is because of the smallest area subjected into mining and carvings activities.

In terms of AGC removals, timber and fire accounts for the highest AGC removals. This may be explained by the large trees removals that comprises of the largest biomass. According to [33], large trees tend to account for a large proportion of the AGB in mature forests; often between 30 and $40 \%$ of the AGB can be found in trees with diameters greater than $70 \mathrm{~cm}$. Elsewhere in miombo [34], found that most miombo had been heavily disturbed because of local benefits attached to them like dry-season fodder for large livestock populations, fuelwood for domestic use and rural industry and construction materials for farm structures and homes for millions. Higher AGC removals in miombo woodlands due to fire is because of its roles as the management tools. When fire is frequently and uncontrolled, it could kill trees and eventually cause carbon emissions. 
Considering administrative zones, charcoal and timber account for higher AGC removals in the Eastern zone. Conversely, charcoal and firewood collection account for higher number of stem removals in this zone. This is due to the highest charcoal and timber consumption that may be linked to the closeness to Dar es Salaam city. Dar es Salaam, Tanzania's largest city, accounts for more than $50 \%$ of all charcoal consumed in the country [35]. Moreover, higher timber consumption in this zone could be attributed to high demand of timber for furniture and infrastructure development particularly houses in the Dar es Salaam city. Dar es Salaam is the primary destination of timber and timber products (including all round and sawn timber) and accounting for $87 \%$ of timber felled in southeast Tanzania [36]. Other important domestic markets of timber and wood products from the zone are Zanzibar, Mafia and Arusha [25]. Shifting cultivation and charcoal account for the largest number of stems and AGC removals in the northern zone probably due to intensification of shifting cultivation. In the lake zone, fire, firewood collection and pole account for the large stems and AGC removals probably due to heavily dependence trees for cooking energy and constructions purposes. Furthermore, presence of dry litter that foster fire occurrence explains the removals due to fire in this zone. The regular fires in the miombo region can, if too frequent or intense, cause mortality of large and small trees and prevent regeneration [37]. Likewise, long-term plot-scale experiments had shown that under annual burning miombo woodland is converted to grassland [38, 37], and that in the absence of fire, miombo starts to form closed canopy forest [37].

Regarding vegetation types, shifting cultivation, charcoal, timber poles, and firewood collection accounted for the highest AGC and number of stems removals in the woodland with scattered woodland. Shifting cultivation type of farming in the country is practiced by more than $70 \%$ of the population. Other scholar [25] found that shifting type of Agriculture is common and practiced for all annual crops grown in Tanzania. The most cited reasons for shifting their plots are; invasion of weeds and evading wild animals. On the other hand, natural death, timber and shifting cultivation accounts for the largest AGC removals in the closed woodland. Natural death is more prominent in this vegetation probably because protection forest and wildlife area comprises most of this vegetation where by no harvesting is allowed. Regarding timber, most of the timber is removed illegally.

In terms of ownership types, fire, timber charcoal and natural death account for higher number of stems removals in all the categories of ownership. This may be attributed to population growth and inadequate presence of alternative sources of energy for cooking and construction purpose that ultimately forces people to heavily depend on charcoal and timber. Irrespective of the fact that, forest under general land is almost open access in which free movement of people take products [39], its contribution to the total removals is low as opposed to private and village land. On the other hand, shifting cultivation accounts for the highest AGC removals in the shifting Agriculture and Agricultural land probably because shifting cultivation type of agriculture characterize the ownership types.

Considering land use types that miombo woodlands falls, it was revealed that shifting cultivation and charcoal account for the highest number of stem removals in grazing and shifting cultivation land. This is because large numbers of stems are removed during land preparation in the shifting cultivation. Likewise, charcoal making and firewood collection characterize the land. Furthermore, natural death, 
poles, charcoal and firewood collection causes more stems cut in the production forest, protection forest and wildlife reserves land. This is much explained by the nature of the ownership types and the large dependence of charcoal and firewood for cooking energy while poles for construction purposes. In contrast, AGC removals that ultimately ends up into carbon emissions are driven by charcoal, natural death, shifting cultivation, poles, timber, fire and firewood collection in all land use types. This may be attributed by population growth that demand more products from the woodlands and climate change impacts that naturally kills trees through eruption of diseases and drought. Moreover, economic growth based on the export of primary commodities and an increasing demand for timber and agricultural products in a globalizing economy are critical reasons behind carbon emissions.

\section{Conclusion}

AGC removals in miombo woodlands of Mainland Tanzania are caused by a range of drivers that lead to varying levels of carbon emissions. The results revealed that charcoal, timber, shifting cultivation, fire, firewood collections, poles and natural death are the prominent main drivers of AGC removals in mainland Tanzania. Interestingly, results also revealed that although charcoal, shifting cultivation and fuelwood drive more tree removals and hence jeopardizes future carbon sink its share to carbon removals is minimal as compared to timber and natural death that account for higher AGC removals. For the purpose of reducing emissions emanating from AGC removals and by considering national circumstances, all drivers should be managed although the management intensity and priorities should consider the significance contribution of AGC emissions by timber, fire, charcoal, shifting cultivation, and natural death in the entire miombo woodlands and its subsequently categories. This would contribute to creation of considerable carbon sink as well as ensure persistent potential for the miombo woodlands to store carbon thus contributing to the REDD + process in Tanzania. Moreover, this kind of study need to be conducted in other vegetation types like Montane and Mangrove forest in Tanzania. On the other hand, since NAFORMA provide national picture on drivers and their variation on AGC removals, we recommend site specific studies be conducted to bring information that would be used to devise appropriate strategies to deal with drivers in their order of contribution to AGC removals in the local settings. Additionally tree planting for timber and energy should be encouraged as mitigating measure.

\section{Methods}

\section{Study Area Description}

The study involved the entire miombo woodlands of mainland Tanzania that covers about 44.7 million ha (Table 1). Vast areas of miombo woodlands falls under the village lands ownership, which lack proper management institution [40]. Depending on altitude and latitude, mainland Tanzania is characterised by both tropical and subtropical climates. The mean annual rainfall varies from below 500 to over $2000 \mathrm{~mm}$ per annum. The rainfall for the large part of the country is bimodal with short rains from October to December and long rains from March to May. The weather conditions of the country may be divided into 
a hot dry season from mid-August to the end of October, a hot wet season from November to the beginning of April and a relatively cool dry season from April to mid-August.

\section{Data Collection \\ Sampling design}

The data used for the assessment of drivers and their influence on variation of AGC removals presented in this paper were collected by NAFORMA [22]. Systematic double sampling for stratification with optimal allocation of individual plots in cluster was sampling design of the NAFORMA(Fig. 2).The design was chosen after sampling simulations to reduce uncertainty of estimates under given budget constraints. The detail of the planning of this design and other uncertainties are given in $[42,22,2,3]$

\section{Data acquisition}

All stumps with diameter $\geq 5 \mathrm{~cm}$ within the circular plot radius of $15 \mathrm{~m}$ were measured for diameter and height using calliper or measuring tape. In addition, age, name and end uses to which the removed trees were put into were identified. The details on how age of the stumps and end uses of the removed trees were decided are given in [43,3]. For the purpose of the present study, all plots that were surveyed for stumps measurement were extracted from NAFORMA database. A total 7323 stumps from 16803 plots were extracted.

\section{Data analysis}

\section{Analysis of drivers of aboveground carbon removals}

To obtain the drivers of AGC removals, the identified trees with their corresponding drivers for their removals were listed. The drivers were sorted alphabetically in order to identify total number of drivers responsible for removals in miombo woodlands. Those drivers that were similar like removals due to firewood collection for domestic and industrial use were regarded as firewood collection.

\section{Drivers and their influence on aboveground carbon removals}

We included multiple drivers identified (11 drivers) in the analysis, so that the interrelationships between the drivers and AGB removed could be accounted. To define the influence of each driver on AGC removals, AGB removed per tree was estimated using allometric equation that estimates tree biomass from the remaining stump [7]. The estimated individual tree AGB removal in its corresponding driver was divided by age of the stump to get the rate of AGB removals per year. AGB removals per year per tree was summed up and expressed on per plot basis. Since each stratum had unique sampling intensity, it was necessary to calculate expansion factors $(E F)$ for each respective stratum since simple mean of AGB would ignore the nature of the sampling design upon which the data were collected. The $E F$ describes the 
area in which a sample plot represents in each stratum. The details on how the $E F$ factor was calculated are shown in [2,3]. Consequently, AGB plot level values were multiplied by respective $E F$ value corresponding to each stratum. The AGB plot level values were expressed on per hectare (ha). To obtain the influence of each driver on AGC removals, AGB removals per ha values were multiplied by 0.49 as the conversion factor of $A G B$ to $A G C$ [44]. Finally, the $A G C$ and their corresponding drivers were summarized in terms of Zones, miombo vegetation subtypes, Land use and Ownership types.

\section{Abbreviations}

AGC: Aboveground carbon; NAFORMA: National Forest Resources Assessment and Monitoring; GHG: Greenhouse Gas Emissions; AGB: Aboveground biomass; NFI: National Forest Inventory; REDD+: Reduced Emissions from deforestation and forest degradation "plus," the role of conservation, sustainable management of forests, and enhanced carbon stock; TFS: Tanzania Forest Services Agency; IPCC: Intergovernmental Panel for Climate Change.

\section{Declarations}

\section{Ethics approval and consent to participate}

Not applicable

\section{Consent for publication}

Not applicable

\section{Funding}

No financial support was provided regarding the preparation of this manuscript

\section{Availability of data and materials}

All authors declare that the datasets used in this study are available upon request from the Tanzania Forest Service, Ministry of Natural Resources and Tourism Tanzania.

\section{Authors' contributions}

BJM has been involved in designing the study, drafting the manuscript, data analysis and write up: WAM performed analysis and revised the manuscript: EFN and REM made substantial contributions to conception and revising the manuscript. REM, EFN, WAM have given final approval of the version to be published. All authors read and approved the final manuscript.

\section{Acknowledgments}


The authors would like to thank the Tanzania forest services (TFS) of the Ministry of Natural Resources and Tourism (MNRT) in Tanzania for giving us a permit to use NAFORMA data.

\section{Competing interests}

The authors declare that they have no competing interests

\section{References}

1. United Republic of Tanzania. Tanzania's Forest Reference Emission Level passed by the United Nations Framework Convention on Climate Change (FREL). Ministry of natural resources and tourism. Tanzania: Dar es Salaam; 2018. 42 pp.

2. Mauya EW, Mugasha WA, Njana MA, Zahabu E, Malimbwi RE. Carbon stocks for different land cover types in Mainland Tanzania. Carbon Balance Management. 2019;14:4.

3. Manyanda BJ, Nzunda EF, Mugasha WA, Malimbwi RE Estimates of volume and carbon stock removals in Miombo woodlands of mainland Tanzania. Inter J For Res. 2020; 2020: 10 pp.

4. United Republic of Tanzania

National Bureau of statistics population and housing census 2002 Accessed on 24 December 2019.

United Republic of Tanzania. National Bureau of statistics population and housing census 2002. http://Tanzania.go.tz/census/region. Accessed on 24 December 2019.

5. FAO. Global Forest Resources Assessment, Main Report. Forestry Paper no. 163, Rome, Italy 2010.

6. WWF. Importance of Forests. 2016.

http://wwf.panda.org/about_our_earth/deforestation/importance_forests/ Accessed on 16 October 2019.

7. Manyanda BJ, Mugasha WA, Nzunda EF, Malimbwi RE Biomass and volumes models based on stump diameter for assessing forest degradation in Miombo woodlands in Tanzania. Inter J For Res. 2019; 2019: 15 pp.

8. IPCC. Climate change Impacts, Adaptation and Vulnerability. Summary for policy makers. IPCC AR4, 2007.

9. Asner GP, Knapp DE, Broadbent EN, Oliveira PJC, Keller M, Silva JN. Selective logging in the Brazilian Amazon. Science. 2005;310:480-2.

10. Marklund LG, Schoene D. Global assessment of growing stock, biomass and carbon stock. Global forest resources assessment. Food and Agriculture Organization of the United Nations; Rome Italy.2006.

11. Geist $\mathrm{H}$, Lambin E. Proximate causes and underlying driving forces of tropical deforestation. Bio Science. 2002;52:143-50.

12. Gallardo AR. The influence of markets and culture on the use of native forests in the south of Chile. A thesis submitted in partial fulfillment of the requirements for the degree of doctor of philosophy in 
the faculty of graduate and postdoctoral studies (forestry), the University of British Columbia. 2017; $106 p$.

13. Schoene D, Killmann H, Von Lu PKE, Loychewilkie M Definitional issues related to Reducing Emissions from Deforestation in Developing Countries. Forests and Climate Change Working Paper no.5. Rome Italy. 2007.

14. Hosonuma N, Herold M, DeSy V, De Fries RS, Brockhaus M, Verchot L, Angelsen A, Romijn E. An Assessment of Deforestation and Forest Degradation Drivers in Developing Countries. Environmental Research Letters. 2012;7:4009.

15. Agriculture IPCC, Forestry and other Land use. In: Eggleston HS, Buendia L, Miwa K, Ngara T, Tanabe $\mathrm{K}$, eds. IPCC guidelines for national greenhouse gas inventories. Prepared by the national greenhouse gas inventories programme, institute for global environmental strategies (iges), Hayama, Japan. 2006.

16. FAO. Towards Defining Degradation. Forest Resources Assessment Working Paper no.154, Rome, Italy. 2009; $11 \mathrm{p}$.

17. Lambin EF, Geist HJ, Lepers E. Dynamics of land-use and land-cover change in tropical regions. Annual Rev Environ Resource. 2003;28:205-41 pp.

18. Kissinger G, Herold M, De Sy V Drivers of Deforestation and Forest Degradation: A synthesis Report for REDD + Policymakers. Lexeme Consulting, Vancouver Canada. 2012; 45 pp.

19. Chave J, Condit R, Lao S, Caspersen JP, Foster RB, Hubbell SP. Spatial and temporal variation of biomass in a tropical forest: results from a large census plot in Panama. J Ecol. 2003;91:240-52.

20. Baker T, Phillips $\mathrm{O}$, Malhi Y. Variations in wood density determines spatial patterns in Amazonian forest biomass. Glob Change Biol. 2004;10:545-62.

21. Houghton R. Aboveground Forest Biomass and the Global Carbon Balance. Glob Change Biol. 2005;11:945-58.

22. MNRT. National Forest Resources Monitoring and Assessment of Tanzania mainland (NAFORMA). Main results. 2015, 106p.

23. Kessy JF, Nsokko E, Kaswamila E, kimaro F. Analysis of drivers and agents of deforestation and forest degradation in Masito forests, kigoma, Tanzania. International Journal of Asian Social Science. 2016;6(2):93-107 pp.

24. Makunga EJ, Misana BS. The Extent and Drivers of Deforestation and Forest Degradation in MasitoUgalla Ecosystem, Kigoma Region, Tanzania. Open Journal of Forestry. 2017;7:285-305 pp.

25. Miya M, Ball SMJ, Nelson FD Drivers of Deforestation and Forest Degradation in Kilwa District. Mpingo Conservation and Development Initiative. 2012, 34p.

26. the Forestry Development Center, University of the Philippines Los Banos - College of Forestry and Natural Resources

Carandang AP, Bugayong LA, Dolom PC, Garcia LN, Villanueva MB, Espirit NO. and the Forestry Development Center, University of the Philippines Los Banos - College of Forestry and Natural Resources. Analysis of Key Drivers of deforestation and Forest Degradation in the Philippines. 
Deutsche Gesellschaft für Internationale Zusammenarbeit (GIZ) GmbH, Manila, Philippines. 2013; $128 p$.

27. FAO. Emerging approaches to Forest Reference Emission Levels and/ or Forest Reference Levels for REDD+. The UNREDD Programme 2014. [http://www.unred d.net/index.phpop tion = com_docma n\&task = doc_downl oad\&gid $=13469$ \&ltemi $d=53$ ] Accessed 20 December 2019.

28. Sawe E. An overview of charcoal industry in Tanzania-issues and challenges; prepared for the national R\&D committee on industry and energy. Tanzania Traditional Energy Development and Environment Organization (TaTEDO), Dar es Salaam.2004.

29. Mwampamba TH. (2007). Has the Wood Fuel Crisis Returned? Urban Charcoal Consumption in Tanzania and Its Implications to Present and Future Forest Availability 2019.

[http://www.charcoalproject.org/wp-content/uploads/2011/01/Mwampamba-2007-CharcoalTanzania] Accessed on 24 May 2019.

30. Angonese GJ, Grau G. Assessment of swaps and persistence in land cover changes in a subtropical periurban region, NM Argentina. Landscape urban Planning. 2014;127:83-93.

31. Ouedraogo I, Mbow C, Balinga M, Neufeldt H. .Transitions in Land Use Architecture under multiple Human Driving Forces in a Semi - Arid Zone. Land. 2015;4:560-77.

32. United Republic of Tanzania. National Forest Policy. Ministry of Natural Resources and Tourism, Dar es Salaam, Tanzania.1998, 59p.

33. Brown S, Schroeder P, Birdsey R. Aboveground biomass distribution of US eastern hardwood forests and the use of large trees as an indicator of forest development. For Ecol Manage. 1997;96(12):37-47.

34. Dewees PA, Bruce M, Campbell YK, Almeida S, Angelsen ABC. A. and Wunder S. Managing the Miombo Woodlands of Southern Africa: Policies, Incentives and Options for the Rural Poor. Journal of Natural Resources Policy Research. 2010;2(1):57-73.

35. Ahrends A, Burgess ND, Milledge SAH, Bulling MT, Fisher B, Smart JCR, Clarke GP, Mhoro B,E, Lewis SL. Predictable waves of sequential forest degradation and biodiversity loss spreading from an African city. PNAS. 2010;107(33):14556-61.

36. Milledge SAH, Elibariki R The status of logging in Southern Tanzania. A technical report submitted to the WWF East Africa Coastal Forests Ecoregion Programme. Project number: IP0002/2005. TRAFFIC East/Southern Africa. 2005.

37. Ryan CM, Williams M. How does fire intensity and frequency affect miombo woodland tree populations and biomass? Ecol Appl. 2011;21:48-60.

38. Furley PA, Rees RM, Ryan CM. (2008). Savanna burning and the assessment of long-term fire experiments with particular reference to Zimbabwe. Progress In Physical Geography. 2008; 32, 611634.

39. Mwakalobo ABS, Kajembe GC, Silayo DS, Nzunda EF, Zahabu E, Maliondo S, Kimaro, D.N. REDD + and sustainable development- perspective from Tanzania. REDD working papers IIED, London. 2011. 
40. Malimbwi R, Eid T, Chamshama S. Allometric tree biomass and volume models in Tanzania. Morogoro: E \& D publishing on behalf of Department of Forest Resources Assessment and Management, Sokoine University of Agriculture; 2018. 129p.

41. Mauya EW, Mugasha WA, Zahabu E, Ole M, Bollandså S, Eid T. (2014). Models for estimation of tree volume in the miombo woodlands of Tanzania. Southern Forests: Journal of Forest Science. 2014; 10: $1-11$.

42. Tomppo E, Malimbwi R, Katila M, Mäkisara K, Henttonen HM, Chamuya N, Zahabu E, Otieno J. A sampling design for a large area forest inventory: A case of Tanzania. Journal of Forest Research. 2014;44:931-48.

43. United Republic of Tanzania. NAFORMA field manual Biophysical survey. Forestry and Beekeeping Division, Ministry of Natural Resources and Tourism. Dar es Salaam Tanzania. 2010, 96p.

44. MacDicken KG. (1997). A Guide to Monitoring Carbon Storage in Forestry and Agroforestry Projects. Report No. 1611. International Institute for Agricultural Development, Arlington, USA. 1997, 87 pp.

\section{Figures}




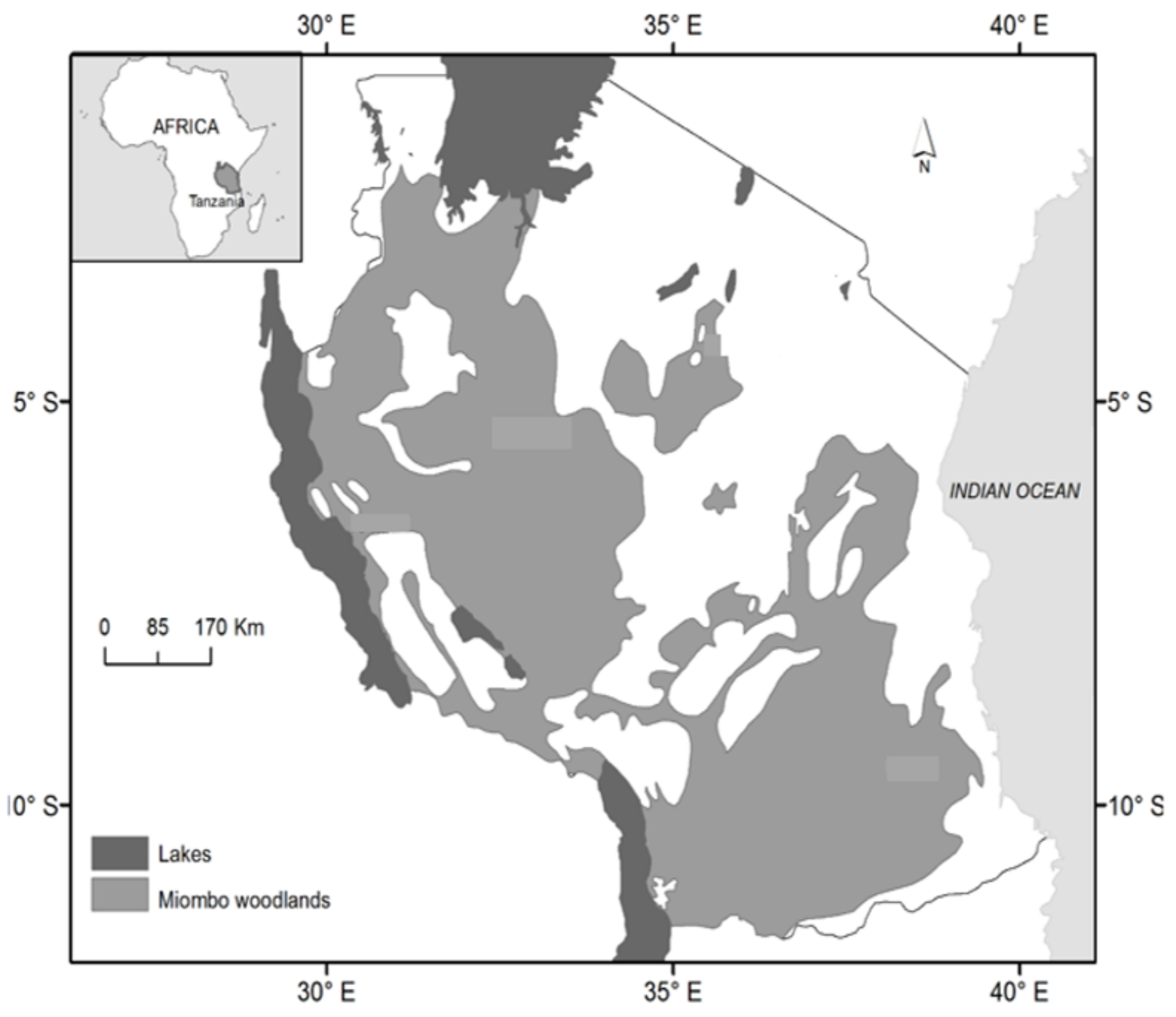

Figure 1

A map of Mainland Tanzania showing Miombo woodlands (modified from [41]) 


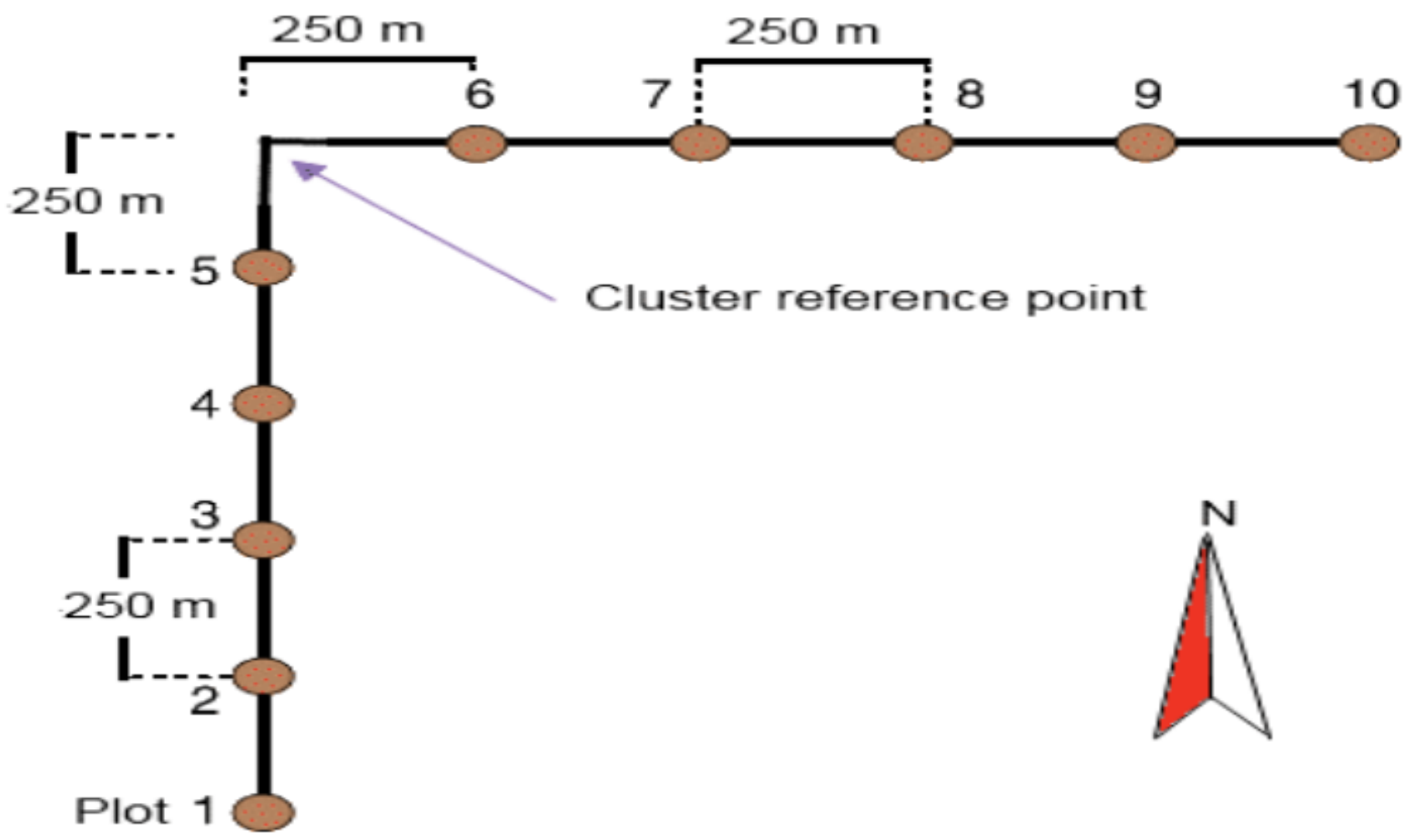

Figure 2

Cluster design (source: [22]) 